\title{
Identification and functional characterization of a rice NAC gene involved in the regulation of leaf senescence
}

Yong Zhou', Weifeng Huang ${ }^{1}$, Li Liư ${ }^{2}$,Taiyu Chen ${ }^{1}$, Fei Zhou ${ }^{1}$ and Yongjun Lin ${ }^{1 *}$

\begin{abstract}
Background: As the final stage of leaf development, leaf senescence may cause the decline of photosynthesis and gradual reduction of carbon assimilation, which makes it a possible limiting factor for crop yield. NACs are plantspecific transcription factors and some NACs have been confirmed to play important roles in regulating leaf senescence.

Results: In this study, we reported a member of the NAC transcription factor family named OSNAP whose expression is associated with leaf senescence, and investigated its preliminary function during the process of leaf senescence. The results of qRT-PCR showed that the OsNAP transcripts were accumulated gradually in response to leaf senescence and treatment with methyl jasmonic acid (MeJA). A subcellular localization assay indicated that OsNAP is a nuclear-localized protein. Yeast one-hybrid experiments indicated that OsNAP can bind the NAC recognition site (NACRS)-like sequence. OsNAP-overexpressing transgenic plants displayed an accelerated leaf senescence phenotype at the grain-filling stage, which might be caused by the elevated JA levels and the increased expression of the JA biosynthesis-related genes LOX2 and AOC1, and showed enhanced tolerance ability to MeJA treatment at the seedling stage. Nevertheless, the leaf senescence process was delayed in OsNAP RNAi transgenic plants with a dramatic drop in JA levels and with decreased expression levels of the JA biosynthesisrelated genes $A O S 2, A O C 1$ and OPR7.
\end{abstract}

Conclusions: These results suggest that OsNAP acts as a positive regulator of leaf senescence and this regulation may occur via the JA pathway.

Keywords: Chlorophyll, Leaf senescence, NAC, JA, Rice (Oryza sativa L.)

\section{Background}

Leaf senescence is complex in that it involves many highly organized molecular and cellular processes such as the disintegration of chloroplast, down-regulation of photosynthesis, degradation of nucleic acid, protein, and lipid and recycling of nutrients. And as the final stage of leaf development, leaf senescence eventually leads to leaf death, which is controlled by both internal and external factors. The internal factors include age, phytohormone levels and developmental processes, and the external factors mainly comprise environmental/biological stresses such as extreme

\footnotetext{
* Correspondence: yongjunlin@mail.hzau.edu.cn

${ }^{1}$ National Key Laboratory of Crop Genetic Improvement and National Centre of Plant Gene Research, Huazhong Agricultural University, Wuhan, 430070, China

Full list of author information is available at the end of the article
}

temperature, shading, drought, wounding, nutrient limitation, pathogen attack and oxidative stress by UV-B irradiation and ozone [1-3].

NACs (NAM, ATAF and $\underline{\mathrm{CUC}}$ ) are plant-specific transcription factors and are widely found in plants. It has been reported that many NACs show enhanced expression during dark-induced and natural leaf senescence in Arabidopsis [4,5], and may play a central role in mediating leaf senescence. The senescence-controlling NAC gene $N A M-B 1$ was also reported to be associated with the contents of grain protein, zinc, and iron in wheat [6]. ANAC092/AtNAC2/ORE1, whose expression correlates with senescence, positively regulates aging-induced cell death in Arabidopsis leaves [7]. The oresara1 (ore1) mutant, which lacks the functional ANAC092/AtNAC2/ORE1 gene, displays a delayed leaf senescence phenotype [8]. 
An ABA-responsive NAC transcription factor VNDINTERACTING2 (VNI2), whose expression shows a leaf aging or leaf longevity-dependent expression pattern, may mediate the crosstalk between the salt stress response and the leaf aging process [9]. AtNAP is strongly up-regulated during leaf senescence in Arabidopsis, and atnap null mutants show a delayed leaf senescence phenotype, whereas the inducible overexpression of AtNAP causes precocious leaf senescence [10]. Recent studies have shown that AtNAP can bind to the promoter region of SENESCENCE-ASSOCIATED GENE113 (SAG113) to form a ABA-AtNAP-SAG113 protein phosphastase $2 \mathrm{C}$ regulatory chain for controlling stomatal movement and water loss in senescing Arabidopsis leaves [11,12]. Many NAC transcription factors can be induced by leaf senescence, but their particular roles in leaf senescence still remain largely unknown.

Many studies have revealed that JA and its derivatives play important roles in regulating the response to leaf senescence in plants. MeJA and its precursor JA were first isolated in oat and shown to promote senescence in detached oat leaves, suggesting that jasmonates might serve as powerful promoters to induce plant senescence [13]. JA-induced leaf senescence is accompanied by the increased expression of several enzymes involved in JA biosynthesis and the decreased expression of the genes involved in photosynthesis [14-16]. In addition, JA can activate the expression of many senescence-regulated genes such as AtWRKY6 [17], OsAkaGal [18] and ESR/ ESP [19], which play major roles in leaf senescence. Leaf senescence and MeJA can induce the expression of OsAkaGal, which encodes a chloroplast alkaline $\alpha$ galactosidase involved in the degradation of digalactosyl diacylglycerol during leaf senescence in rice $[18,20]$. Exogenous application of JA to attached and detached leaves promotes leaf senescence in Arabidopsis but does not induce leaf senescence in the JA-insensitive mutant coil, suggesting that the JA-signaling pathway is required for JA to promote leaf senescence [14]. Furthermore, JA promotes $\mathrm{H}_{2} \mathrm{O}_{2}$ accumulation in the leaves of JA-sensitive cultivar TN1 seedlings to accelerate the process of senescence but not in the leaves of JA-insensitive cultivar TNG67 [21]. Many JA and senescence-regulated genes have been identified, yet how the crosstalk between JA signaling and senescence occurs remains to be thoroughly understood.

In this study, we isolated and characterized the JAinduced senescence-associated gene OsNAP, which encodes a NAC transcription factor in rice. We generated the OsNAP overexpression and RNAi lines and analyzed the leaf senescence process of them. We also determined the endogenous JA levels and checked the expression of the genes encoding the enzymes of the JA biosynthetic pathway in the OsNAP transgenic lines and wild-type plants. Studies of these lines indicated that OsNAP acts as a positive regulator of the JA pathway to mediate the leaf senescence process in rice.

\section{Results}

\section{Characterization of OsNAP in rice}

There are 75 predicted NAC proteins [22] and 140 putative NAC or NAC-like proteins (ONAC) in rice [23]. Later computational analyses showed that there are at least 151 OsNAC genes in the rice genome [24]. And phylogenetic analysis showed that there are 13 OsNACs which closely cluster with AtNAP: Os03g21060 (OsNAP), Os12g03040, Os11g03300 (OsNAC10), Os03g60080 (SNAC1/OsNAC9), Os01g60020 (OsNAC4), Os07g12340 (OsNAC3), Os11g08210 (OsNAC5), Os07g37920, Os05g34310, Os07g48450, Os01g01430, Os05g34830 and Os01g66120 (OsNAC6/SNAC2) (Additional file 1: Figure S1). Digital expression profile analysis showed that the expression levels of Os07g37920, Os05g34310, Os07g48450 and Os01g01430 are low and even undetectable in leaves. And other 9 genes are expressed in leaves, but only the transcripts of OsNAP, OsNAC5 and $O s N A C 6 / S N A C 2$ in leaves are up-regulated during leaf senescence [25-27]. However, several studies have demonstrated that OsNAC5 and OsNAC6/SNAC2 are involved in stress tolerance in rice [28-30]. We therefore decided to examine the potential role of OsNAP in the leaf senescence of rice.

The full-length cDNA of OsNAP was isolated for further functional analysis. OsNAP cDNA (Accession number AK243514) encodes a protein with 392 amino acids. Sequence analysis suggested that OsNAP is identical to ONAC058, which belongs to the NAC family in rice [23]. Twelve sequences were obtained from Arabidopsis thaliana, Oryza sativa, Populus trichocarpa, Gossypium hirsutum, Glycine max, Bambusa emeiensis, Brachypodium distachyon, Sorghum bicolor, Hordeum vulgare, and Zea mays by BLASTP search. The resulting phylogenetic relationships showed that each of them contained A-E subdomains (Additional file 1: Figure S2), which is consistent with the results previously reported $[31,32]$.

\section{Subcellular localization of OsNAP}

To determine the subcellular localization of OsNAP, a construct expressing OsNAP linked to GFP under the control of the maize (Zea mays) ubiquitin promoter was transiently transfected into onion cells. The fluorescence was observed only in the nuclei, which was confirmed by DAPI staining (Figure 1A). To further study the localization of OsNAP, Arabidopsis cell protoplasts were co-transformed using 35S::OsNAP-GFP and 35S::Ghd7CFP. As shown in Figure 1B, GFP fluorescence from OsNAP overlapped with the CFP signals from GHD7 


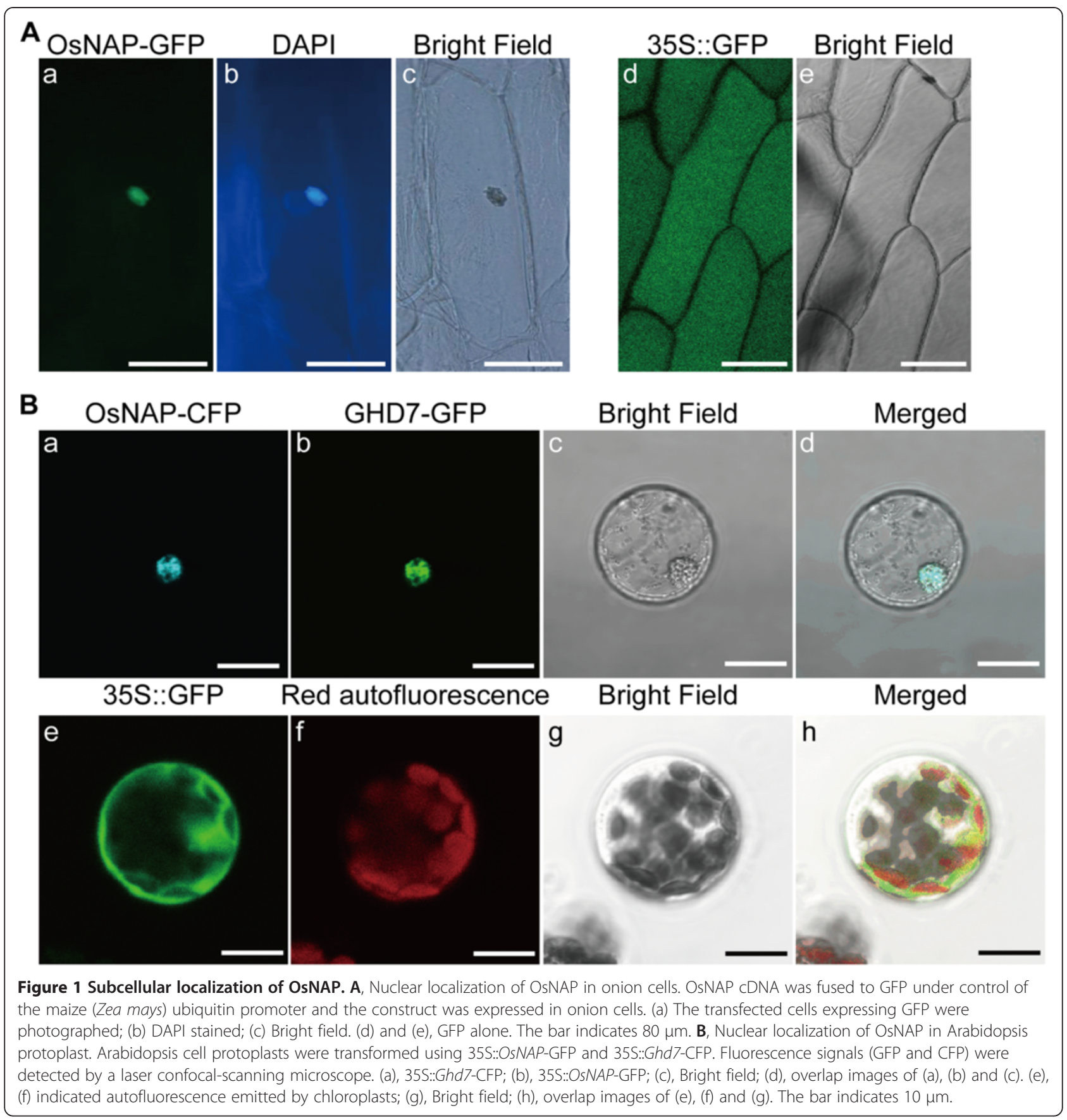

[33], which further confirmed that OsNAP localizes to the nucleus.

\section{Biochemical function of OsNAP in yeast}

Some NAC transcriptional factors bind to the NAC recognition site (NACRS)-like sequence in the OsERD1 (early responsive to drought 1 ) promoter to activate HIS reporter gene expression [30,34-36]. To detect whether OsNAP can bind to this sequence, the ORF of OsNAP was fused to GAL4-AD in the pGADT7 vector and co-transformed with the pHIS2-cis reporter vector [34] into the Y187 yeast strain (Figure 2A). The combinations of pGAD-Rec2-53/ p53HIS and pGAD-OsNAP/p53HIS2 were used as the positive control and negative control, respectively. The result showed that all of the combinations, including pGADOsNAP/pHIS2-cis, the positive and negative control, could grow on the SD/Leu-/Trp-/His- medium without 3-AT. However, when the SD/Leu-/Trp-/His- medium was supplemented with $30 \mathrm{mM} 3-\mathrm{AT}$, the transformed pGADOsNAP/pHIS2-cis and the positive control grew, whereas 


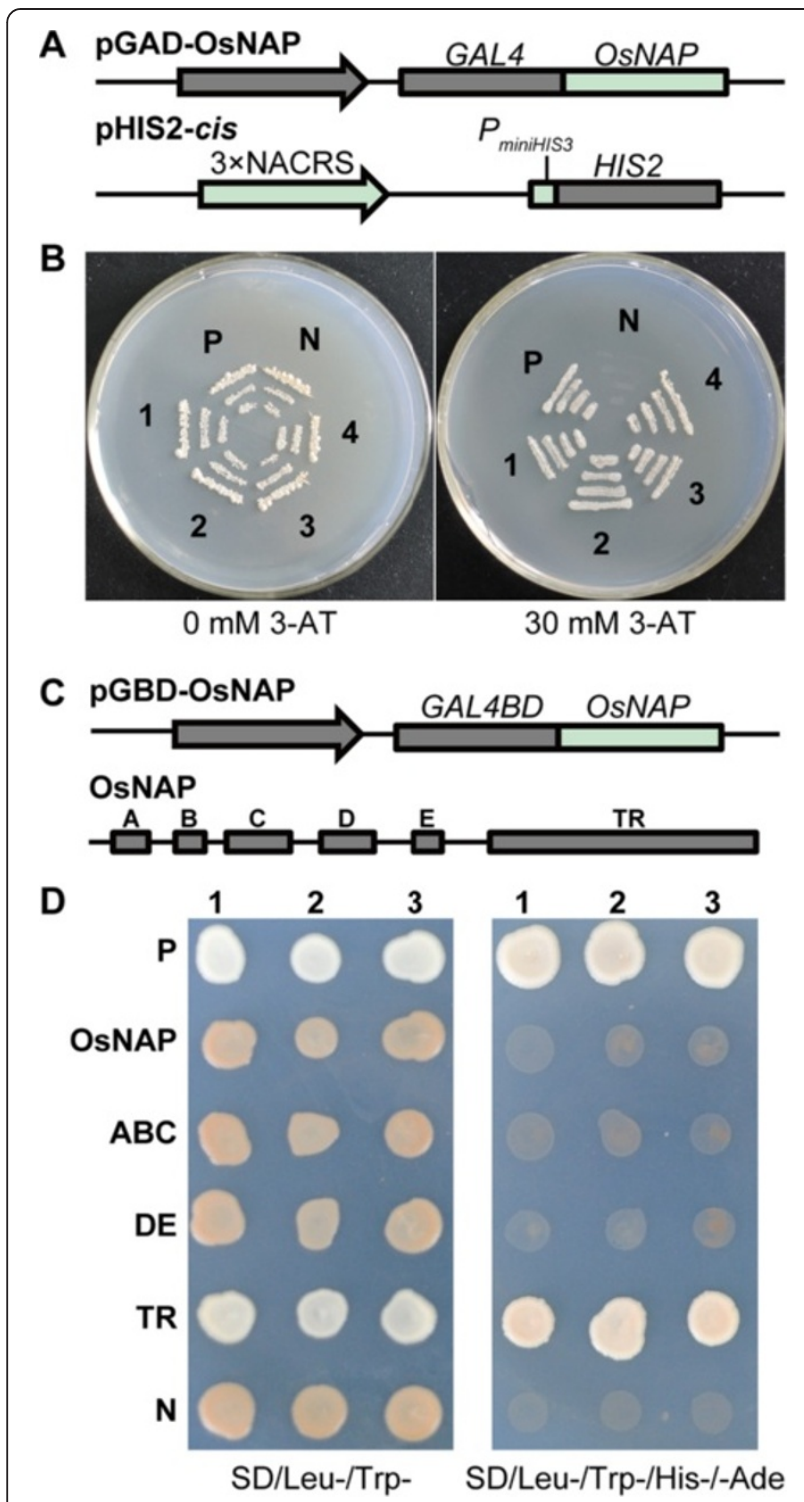

Figure 2 Biochemical function of OsNAP in yeast. A, Constructs for the yeast one-hybrid assay. B, OsNAP can bind to the NACRS-like sequence in yeast. The pGAD-OsNAP plasmid and reporter construct pHIS-cis [34] were cotransformed into the Y187 yeast strain. The transformants were examined by growth performance on SD/Leu-/ Trp-/His- plates with or without $30 \mathrm{mM}$ 3-AT. N, negative control (p53HIS2 plus pGAD-OsNAP); P, positive control (p53HIS plus pGADRec2-53); lables 1-4, four different colonies containing pGAD-OsNAP and pHIS-cis. C, Constructs of transactivational analysis. D, Transactivation assay of OsNAP in yeast. The full-length and partial fragments of OsNAP were ligated into the PGBDKT7 vector and co-transformed into the AH109 yeast strain with $\mathrm{pGADT7}$ to verify the transactivation activity. The transformants were examined by growth performance on SD plates in the absence or presence of histidine and the necessary amino acids. N, negative control (pGBDK7/pGADT7); P, positive control (pHIS53/pGAD-Rec2-53); lables 1-3, three different colonies containing positive control, pGBD-OsNAP/pGADT7, pGBD-OsNAP-ABC/ pGADT7, pGBD-OsNAP-DE/pGADT7, pGBD-OsNAP-TR/pGADT7 and negative control, respectively. the negative control did not (Figure 2B), indicating that OsNAP binds to the NACRS-like sequence in yeast.

Several NACs not only act on gene promoters but also have transactivation activity $[30,34,36]$. To determinate whether OsNAP has activation capacity, we tested the transactivation activity of OsNAP in yeast. The fulllength and partial fragments of OsNAP were fused to the GAL4 DNA binding domain in the pGBKT7 vector and the resultant vectors were co-transformed with pGADT7 into the AH109 yeast strain (Figure 2C). The combinations of pHIS53/pGAD-Rec2-53 and pGBDK7/ pGADT7 were used as the positive control and negative control, respectively. It was observed that all of the cotransformants grew well on the SD/Leu-/Trp- medium, but only the cells transformed with pHIS53/pGADRec2-53 and pGBD-OsNAP-TR/pGADT7 could grow on the SD/Leu-/Trp-/His-/Ade- medium (Figure 2D), suggesting that the transactivation domain of OsNAP is located in the C-terminal transcriptional regulatory (TR) domain of the protein.

\section{Expression pattern of OsNAP}

To determine the expression profile of OsNAP, qRTPCR assays using total RNA samples from flag leaves of different developmental periods were performed. The results showed that the transcript of OsNAP was associated with the senescence of leaves (Figure 3A). To better understand the OsNAP expression pattern, the OsNAP promoter was fused to the GUS reporter gene and introduced into the Zhonghua 11. Immunohistochemical staining of GUS showed that OsNAP was mainly expressed in callus, leaves, sheaths, nodes, internodes and mature seeds (Figure 3B). In leaves, GUS activity increased gradually with leaf senescence (Figure 3B, b-d), which is consistent with the qRT-PCR result (Figure 3A).

In addition, qRT-PCR analysis showed that the expression of OSNAP was continuously increased during MeJA treatment (Figure $3 \mathrm{C}$ ), indicating that the expression of OsNAP was induced by MeJA treatment.

\section{Effect of the overexpression of OsNAP on leaf senescence} at the grain-filling stage

Because strongly up-regulated expression of OsNAP was observed during leaf senescence in rice (Figure 3A, 3B, b-d), we decided to investigate the role of OsNAP in leaf senescence. The OSNAP gene, which was driven by the constitutive cauliflower mosaic virus $35 \mathrm{~S}$ promoter, was introduced into Zhonghua 11 by an Agrobacteriummediated transformation approach. 10 single-copy insertion lines were obtained based on Southern blot analysis (Additional file 1: Figure S3). The result of the Northern blot analysis showed that the expression levels of OsNAP were substantially elevated in these lines (Additional file 1: Figure S4). All the plants with 

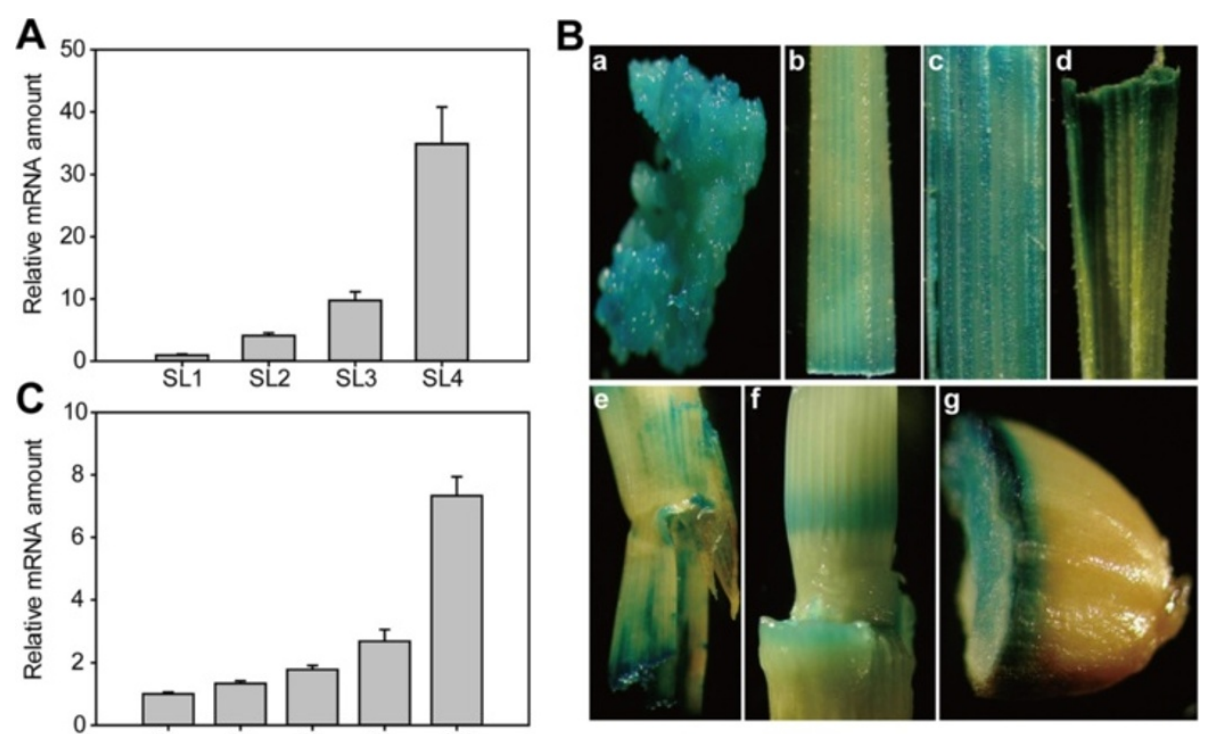

Figure 3 Expression pattern of OsNAP. A, qRT-PCR analysis of OsNAP transcript levels in flag leaves of different development periods in rice. $S L 1$, flag leaves at the booting stage, SL2, flag leaves at the anthesis stage, SL3, flag leaves at the mature stage, SL4, flag leaves at the ripening stage. B, Tissue localization of OsNAP. a, callus; b, young leaf; c, mature leaf; $d$, senescent leaf; e, sheath; $f$, node and internode; $g$, mature seed. C, qRT-PCR analysis of OsNAP transcripts with MeJA treatment at different time points. Roots of Zhonghua 11 plants at the trefoil stage were immersed in nutrition solutions contained $0.1 \mathrm{mM} \mathrm{MeJA}$, and several plants were collected at different points after treatment for RNA isolation.

increased expression levels of OsNAP exhibited visible yellowing phenotype in leaves during the late stages of seed maturation when compared with the WT plants (data not shown). Three OsNAP transgenic lines (line 15, 17 and 19) were selected for further study. The expression levels of OsNAP in these lines were validated using qRTPCR (Figure 4A). No significant difference was observed between the transgenic lines and WT plants during the vegetative and early reproductive stages. However, at 30 $\mathrm{DAH}$, the flag leaves of the high-expressing lines (line 17 and 19) showed an accelerated yellowing phenotype when compared with those of the low-expressing line (line 15) and WT plants (Figure 4B). The yellowing in the leaves became more apparent at $40 \mathrm{DAH}$ (Figure 4C). And the phenotype was correlated with the increasing expression levels of OSNAP (Figure 4D). The time-course changes in the chlorophyll content and net photosynthetic rate of the flag leaves were compared between the OsNAPoverexpressing lines and WT plants after heading. The chlorophyll content and net photosynthetic rate showed no significant differences at around $20 \mathrm{DAH}$ (Figure 4E, 4F). However, the high-expressing lines (line 17 and 19) exhibited more reduction in chlorophyll content and net photosynthetic rate compared with the low-expressing line (line 15) and WT plants at around 30 and $40 \mathrm{DAH}$ (Figure 4E, 4F), suggesting an accelerated leaf senescence phenotype. Taken together, these findings indicate that the overexpression of OsNAP leads to accelerated leaf senescence in rice.
Effect of knock-down of OsNAP on leaf senescence in rice To explore the physiological role of OsNAP in leaf senescence, we generated an OSNAP RNAi construct and introduced it into Zhonghua 11. 10 single-copy insertion lines were verified by Southern blot analysis (Additional file 1: Figure S5). Northern blot analysis showed that the OsNAP transcripts in senescing leaves decreased in most of the transgenic plants (Additional file 1: Figure S6). The leaves of RNAi transgenic lines appeared normal during the vegetative and early reproductive stages, but displayed appreciably higher greenness compared with that of WT plants during the late stages of seed maturation. Two transgenic lines (RNAi-1 and RNAi-2) were selected for further analysis. During the grain-filling stage, an obvious yellowing was observed in the leaves of the WT plants but not in those of the transgenic lines RNAi-1 and RNAi-2 (Figure 5A-C), suggesting that the senescence process was markedly delayed. We further checked the chlorophyll levels in the flag and second top leaves. The chlorophyll levels in the flag and second top leaves of RNAi-1 and RNAi-2 were sharply higher than in those of the WT plants (Figure 5D). qRT-PCR analysis validated that RNAi-1 and RNAi-2 transgenic lines showed a significant reduction in OsNAP transcript levels (Figure 5E). And the greater the reduction in OsNAP transcript levels was, the higher the chlorophyll content became in the leaves of RNAi transgenic lines compared with in those of the WT plants (Figure 5D, $5 \mathrm{E})$, which indicated that the delayed leaf senescence 


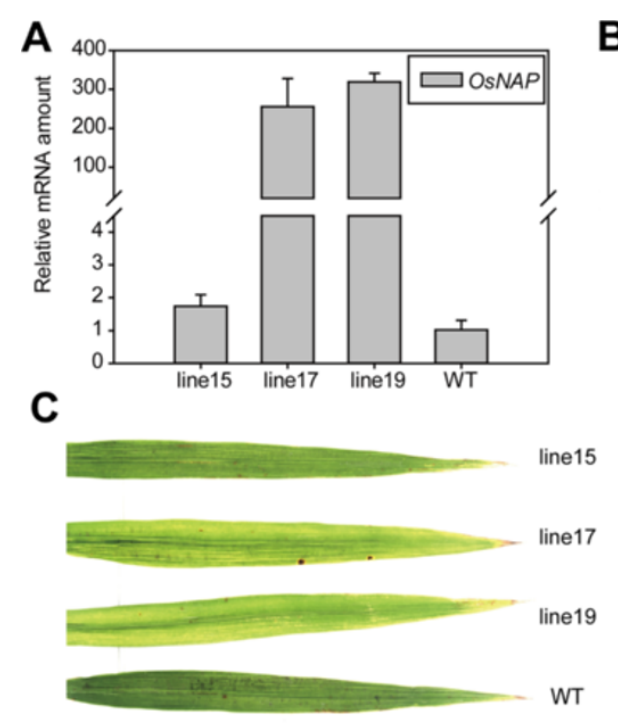

B
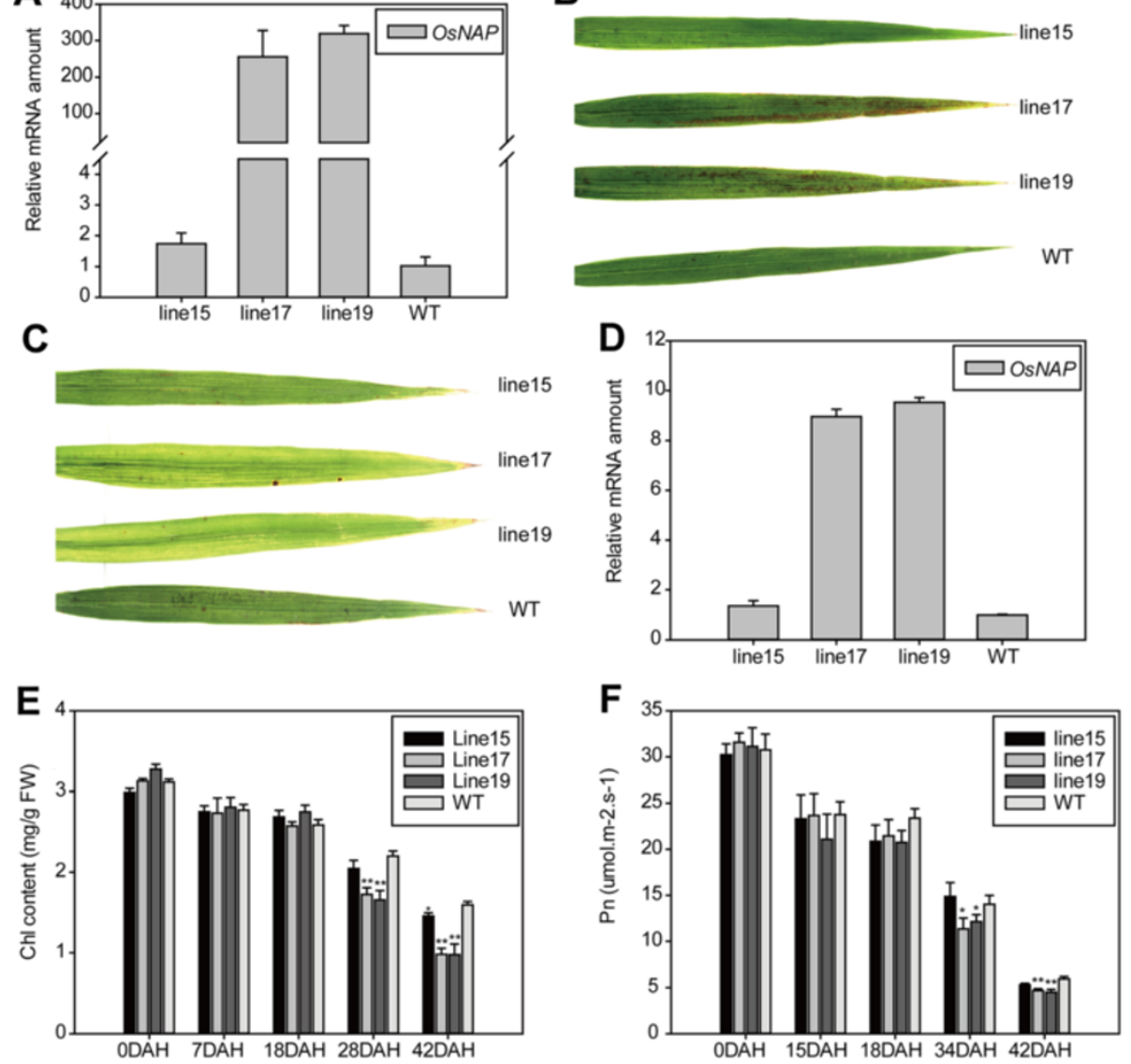

Figure 4 OsNAP overexpression lines showed an accelerated leaf senescence phenotype. A, Expression level of OsNAP in the OsNAP overexpression lines and wild-type plants at the seedling stage. B and C, Photographs of flag leaves in OsNAP overexpression lines and WT plants at 30 (B) and 40 DAH (C). D, Expression level of OsNAP in the OsNAP overexpression lines and wild-type plants at the grain-filling stage. The firststrand CDNAs were prepared using RNAs extracted from the flag leaves of OsNAP overexpression lines and WT plants at the grain-filling stage. Each value represents the mean \pm SD evaluated from three replicates. $\mathbf{E}$ and $\mathbf{F}$, Time course determinations of chlorophyll content and net photosynthetic rate in flag leaves of OsNAP overexpression lines and WT plants after heading. The values are means ( \pm SD) of four replicates.

phenotype was related to the reduction of the expression level of OsNAP. OsDOS acts as a negative regulator of leaf senescence since its expression is down-regulated during leaf senescence [37], which makes OsDOS an ideal molecular marker for leaf senescence in rice. We further analyzed the transcript of OsDOS by qRT-PCR analysis. As shown in Figure 5E, the expression of OsDOS was markedly higher in the leaves of RNAi-1 and RNAi-2 than in those of the WT plants, which further suggested that leaf senescence was remarkably delayed in OsNAP RNAi transgenic plants.

\section{Relation between the senescence phenotype in OsNAP transgenic plants and endogenous JA content}

OsNAP expression was increased gradually by MeJA treatment (Figure $3 \mathrm{C}$ ), implying that OsNAP may be involved in the JA pathway in rice. To assess the function of the OsNAP gene in the JA pathway, a MeJA treatment assay was performed on OsNAP-overexpressing lines and WT plants. There was no significant difference observed in the shoot and root length under normal conditions (Figure 6A). The growth of OsNAP-overexpressing lines and the WT plants was inhibited under MeJA conditions, but the shoot and root length of high OsNAPoverexpressing lines (line 17 and 19) was significantly longer than that of the low-expressing line (line 15) and WT plants (Figure 6B, 6C). However, no difference in shoot and root length was observed between the seedlings of the RNAi lines and the WT plants (data not shown). These findings indicated that the tolerance of OsNAP-overexpressing lines to MeJA treatment was enhanced.

To further explore the function of the OSNAP gene in the JA pathway, we measured the endogenous JA levels in OsNAP transgenic lines and WT plants. As shown in Figure 7A, JA levels were up-regulated in OsNAP-overexpressing transgenic lines, but sharply down-regulated in RNAi lines compared with those in 

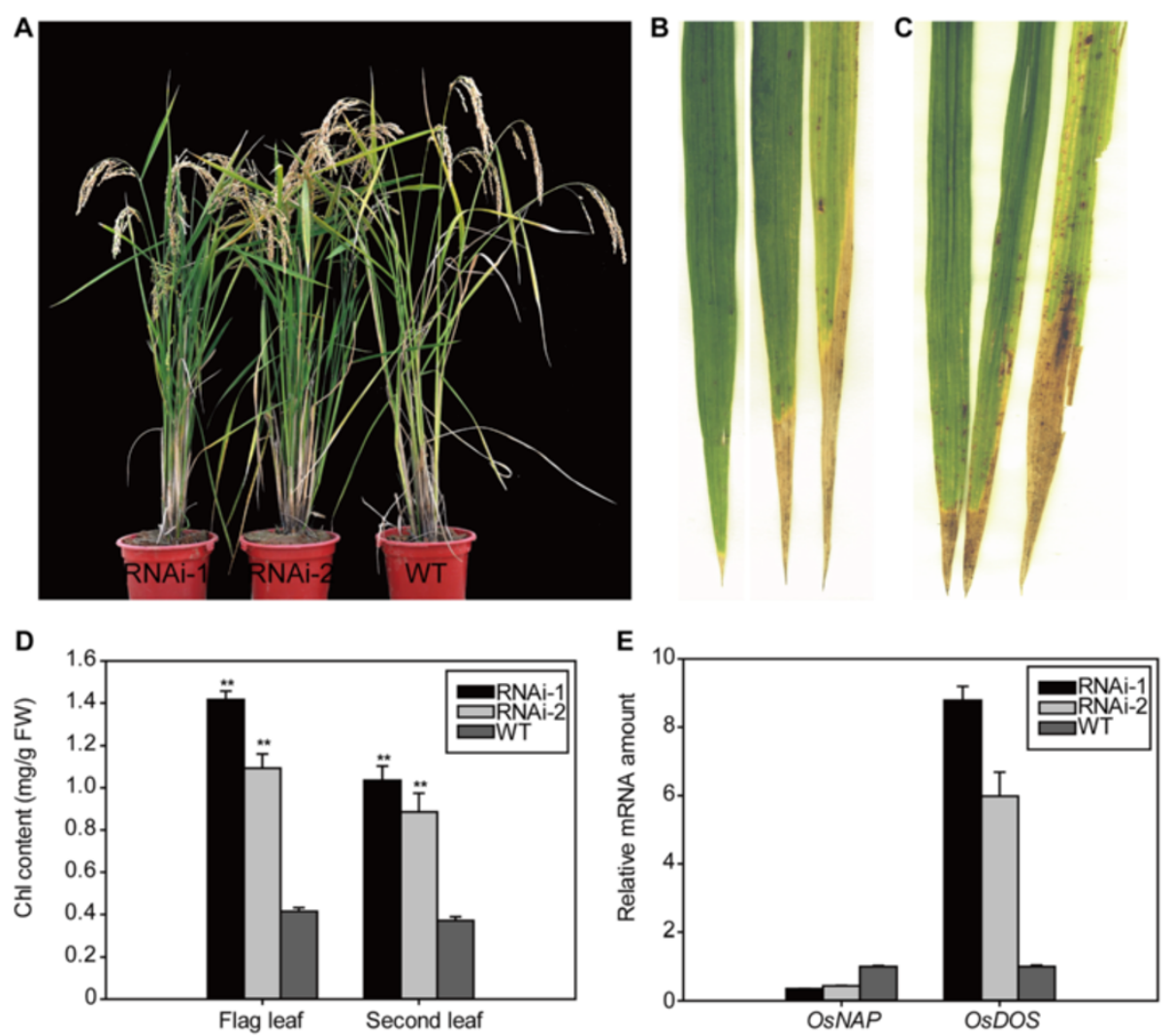

Figure 5 OsNAP RNAi transgenic plants displayed delay of leaf senescence phenotype. A, OsNAP RNAi transgenic lines (RNAi-1 and RNAi-2) and WT plants at 50 DAH. B, flag leaves of RNAi-1, RNAi-2 and WT at 50 DAH. C, Second top leaves of RNAi-1, RNAi-2 and WT at 50 DAH.

D, Content of chlorophyll of flag and second top leaves in RNAi-1, RNAi-2 and WT plants at 50 DAH. Values are means \pm SD $(n=3)$. ${ }^{* *} P<0.01(t$ test). E, qRT-PCR analysis of the expression levels of OsNAP and OsDOS in flag leaves of RNAi-1, RNAi-2 and WT plants at 50 DAH. Each value represents the mean \pm SD evaluated from three replicates.

WT plants whether at the seedling or mature stage. We further analyzed the expression levels of the genes involved in the JA biosynthetic pathway by qRT-PCR in OsNAP transgenic lines and the WT plants. Among these genes, $L O X 2$ and $A O C$ had more significantly increased transcripts in the OsNAP-overexpressing plants than in WT plants (Figure 7B), while the genes that encode enzymes for JA biosynthesis, including AOS2, AOC and OPR7 showed remarkably decreased expression levels in the OsNAP RNAi lines compared with the WT plants (Figure 7C). Therefore, the phenotypes of the OsNAP transgenic plants may at least be partially due to the endogenous JA levels.

\section{Discussion}

Important role of OsNAP in regulating leaf senescence in rice

NAC proteins are unique transcription factors involved in different developmental processes including senescence in plants [38]. Approximately one-fifth of NACs (20/109) are present in the senescence ESTs (expressed sequence tags) in Arabidopsis [5]. About 46\% of the up-regulated genes in ANAC092/AtNAC2/ORE1-overexpressing transgenic plants are senescence-associated genes [39]. These findings suggest that NAC proteins may play crucial roles in senescence.

In this study, we identified a member of the NAC transcription factors OsNAP, which plays an important role in regulating leaf senescence in rice. Firstly, the transcript of $O s N A P$ was up-regulated during natural leaf senescence (Figure 3A, 3B, b-d). Secondly, OsNAP-overexpressing lines displayed an accelerated leaf senescence phenotype and showed a reduced net photosynthetic rate coupled with a decline in chlorophyll content during the reproductive growth stage (Figure 4). In addition, OsNAP RNAi lines showed a markedly delayed leaf senescence, which was confirmed by the higher expression of OsDOS (Figure 5). Finally, as the homolog of AtNAP in rice, OsNAP can restore the delayed leaf senescence phenotype in atnap mutant to a normal WT phenotype [10]. These results suggest that OsNAP is associated with leaf senescence in rice. 


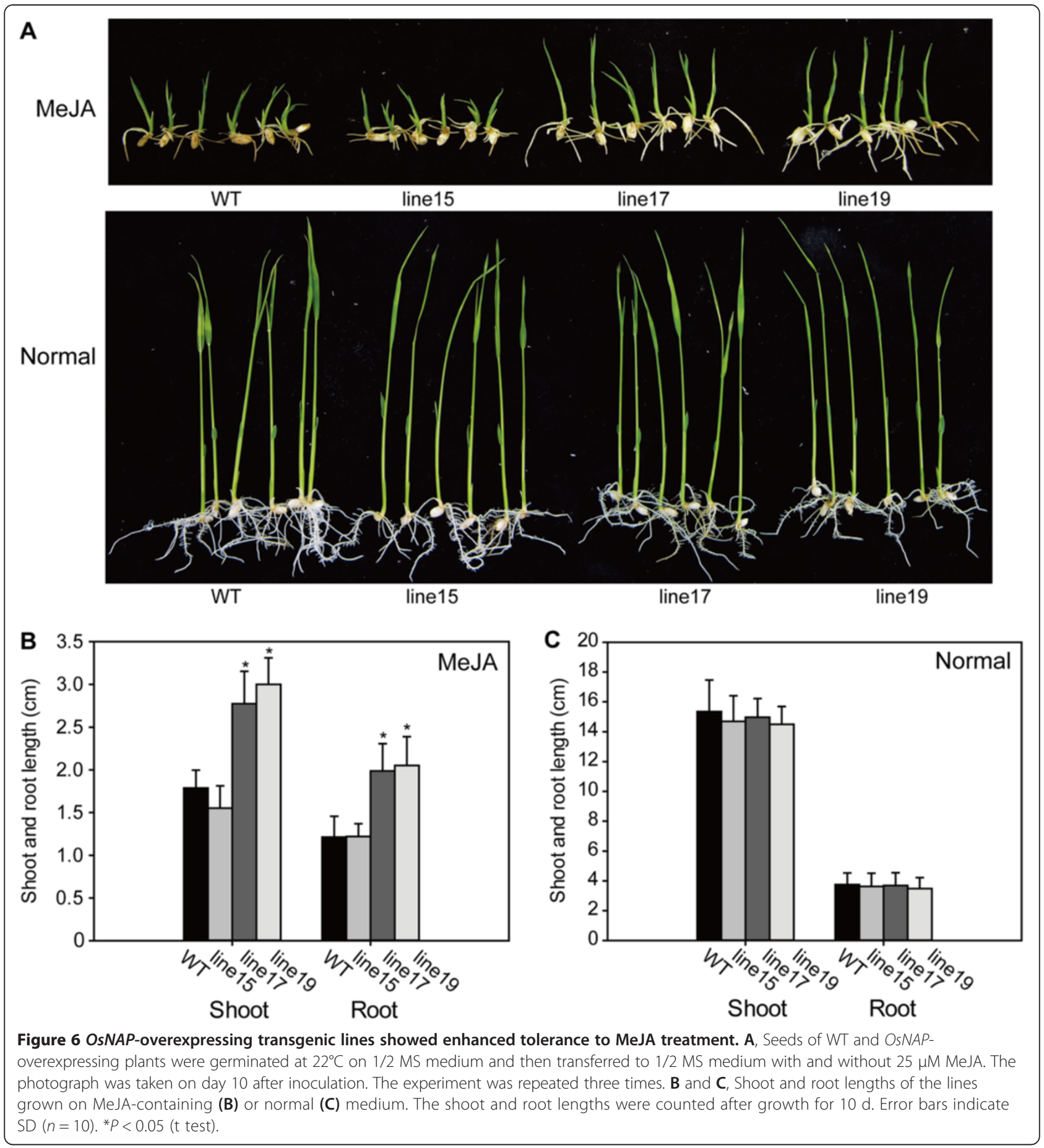

The role of OsNAP in the crosstalk between senescence and the JA pathway

JA and its derivatives are known as endogenous modulators of many physiological processes in plants including senescence $[14,40,41]$. Our analysis revealed that the expression level of OsNAP was up-regulated with MeJA treatment (Figure $3 \mathrm{C}$ ), suggesting that OsNAP may be involved in the JA pathway. There are two important processes in the JA pathway: JA biosynthesis and JA signal transduction $[40,42]$. Accelerated or hindered JA biosynthesis can lead to more rapid or delayed senescence in plants. OsNAP overexpression resulted in accelerated senescence in transgenic rice and it was evident from the decreased chlorophyll content and net photosynthetic rate (Figure 4), which was correlated with accumulation of endogenous JA content and increased transcript levels of 

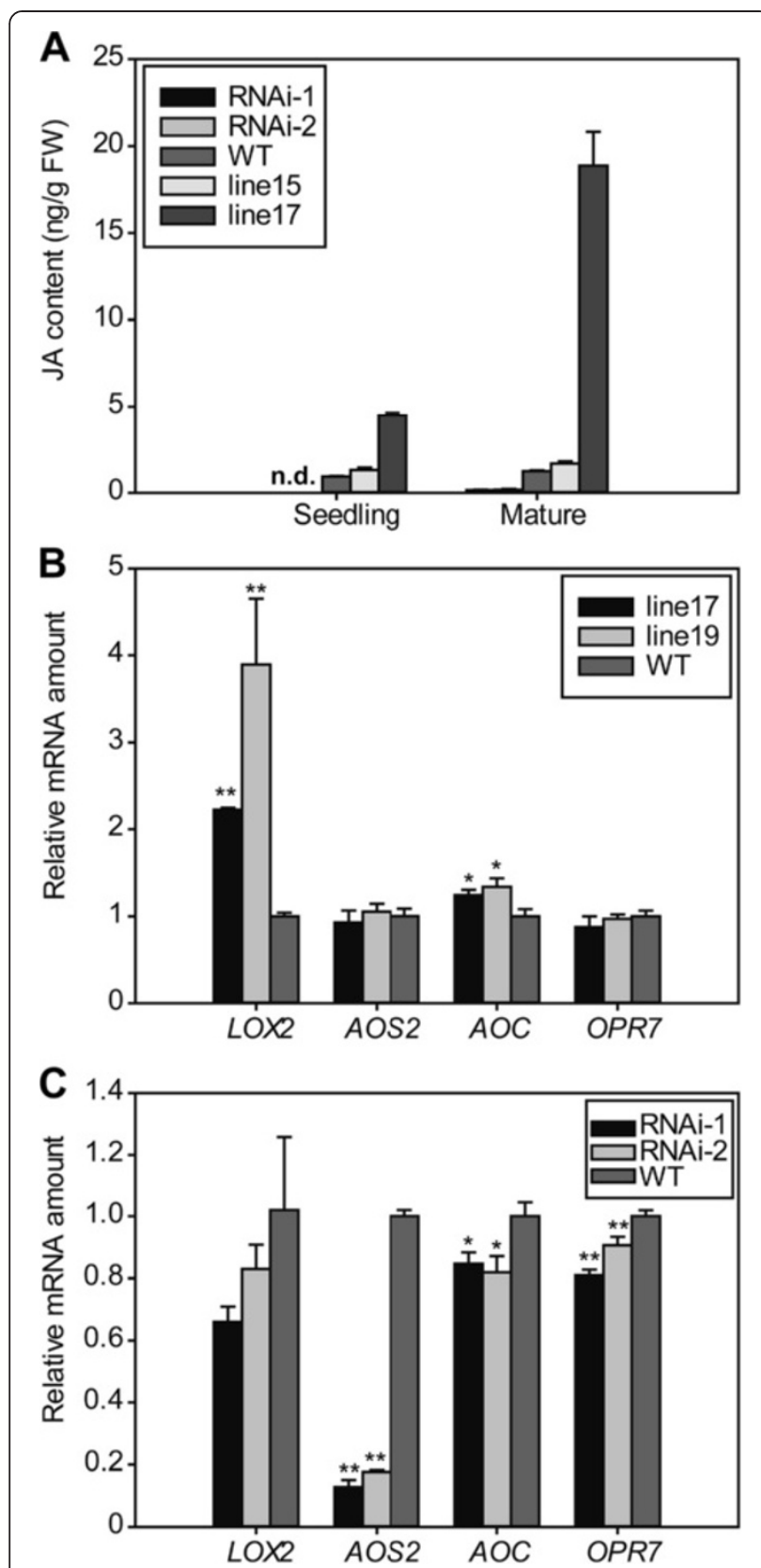

Figure 7 Effects on JA content and JA biosynthetic genes in OsNAP transgenic lines and WT plants. A, Endogenous levels of JA in OSNAP transgenic and WT plants. Leaf samples of OSNAPoverexpressing transgenic lines (line 15 and line 17), OsNAP RNAi lines (RNAi-1 and RNAi-2) and WT plants at seedling and mature stage were used for the analysis. Each point represents a mean (three replicates) $\pm S D$. n.d., no detected. B, qRT-PCR analysis for JA biosynthetic genes in leaves of the OSNAP-overexpressing lines (line 17 and line 19) and WT plants. Transcripts associated with leaves of OsNAP-overexpressing lines and WT plants at the seedling stage were analyzed. C, qRT-PCR analysis for JA biosynthetic genes in leaves of the OsNAP RNAi lines and WT plants at the mature stage. The first-strand CDNAs were prepared using RNAs extracted from the leaves of OSNAP RNAi lines and WT plants at the mature stage. Bars represent mean (3 replicates) \pm standard deviation. ${ }^{*} P<0.05,{ }^{* *} P<0.01$ (t test).
JA biosynthesis genes (Figure 7A, 7B). qRT-PCR results showed that the expression of the JA biosynthesis gene LOX2 was increased 2 to 4 fold in the OsNAPoverexpressing lines compared with in the WT plants (Figure 7B). JA levels were increased during senescence in OsNAP-overexpression transgenic lines and the WT plants, which is in accordance with previous conclusions $[2,4,15]$. On one hand, JA can induce the expression of the key enzymes of chlorophyll breakdown including chlorophyllase [43], promoting the loss of chlorophyll in leaves. On the other hand, JA can also induce the expression of many senescence-associated genes to promote senescence $[14,44]$. Therefore, it is possible that the accelerated leaf senescence observed in OsNAP-overexpressing plants might have resulted from an increase in the endogenous JA levels. In addition, OsNAP RNAi transgenic plants displayed a marked delay of leaf senescence (Figure 5). JA in these lines declined dramatically to non-detectable levels (Figure 7A), and the transcripts of the genes encoding enzymes in the JA biosynthesis pathway, including $A O S 2, A O C$ and OPR7, dropped sharply (Figure 7C). The delayed yellowing during natural senescence in OsNAP RNAi transgenic plants at the grain-filling stage may be due to the reduced JA production. Hence, OsNAP may act as a positive regulator of leaf senescence by regulating the JA biosynthesis pathway.

Besides JA biosynthesis, a block in JA signal transduction can also alter the process of senescence. The mutant coil, whose JA signaling has been impaired, exhibits delayed senescence phenotype [45]. Rubisco activase (RCA) is a COI1-dependent JA-repressed protein and it has been shown that a loss-of-function mutation of $R C A$ leads to typical signs of senescence [46]. OsDOS is a nucleus-localized $\mathrm{CCCH}$-type zinc finger protein in rice that acts as a negative regulator of the JA pathway and senescence process [37]. The transcripts of OsDOS were significantly higher in OsNAP RNAi lines than in the WT plants (Figure 5E), suggesting that the JA signaling pathway was impaired. In addition, MeJA inhibition was significantly lower in the OsNAP high-expressing lines compared with in WT plants, suggesting that the overexpression of OsNAP enhances the resistance to exogenous MeJA in rice (Figure 6). These results indicate that JA signaling pathway is involved in the OsNAPmediated senescence process.

The NAC transcription factors can bind to specific cis-elements of target gene promoters, and can thus regulate gene transcription. For example, the miR319-regulated TCP (TEOSINTE BRANCHED/CYCLOIDEA/PCF) transcription factors regulate the JA biosynthesis gene $L O X 2$, controlling JA content and affecting leaf senescence [47]. And AtNAP can bind to the promoter region of SAG113, which negatively regulates the ABA signaling pathway that 
modulates stomatal movement and water loss in senescing leaves [11]. In the present study, ABA did not have significant effects on OsNAP expression levels (data not shown), while MeJA gradually induced the expression of OsNAP (Figure $3 \mathrm{C}$ ). In addition, OsNAP was able to bind to the NACRS-like sequence (Figure 2A, 2B), and the C-terminal region had transactivation activity in yeast (Figure 2C, 2D). It's worth noting that there were a number of putative NAC recognition sequences (NACRS) and core DNA binding sequences (CDBS) in the $1 \mathrm{~kb}$ region upstream of the JA biosynthesis-related genes (Additional file 1: Table S2). For these reasons, it can be concluded that OsNAP may interact with JA biosynthesis genes directly or indirectly. Further experiments, particularly a screen for the interacting proteins, will be necessary to determine the specific roles of OsNAP in JA signaling and leaf senescence.

\section{Conclusions}

In this article, a NAC transcription factor OsNAP was isolated and characterized in rice, and OsNAP was identified as a key mediator between the JA pathway and leaf senescence. In addition, the expression of OsNAP was detected in the callus, sheath, mature seed, node and internode besides the leaves (Figure 3B), suggesting that OsNAP plays important roles in diverse biologic processes in rice. Nevertheless, further analyses are required to clarify the specific roles of OsNAP in rice.

\section{Methods}

\section{Plant materials and growth conditions}

Zhonghua 11 (Oryza Sativa L. ssp. Japonica cv. Zhonghua 11) was used for this study. Wild-type and OsNAP transgenic plants were planted in the field of Huazhong Agricultural University (Wuhan, China).

To check the OsNAP expression during MeJA treatment, Zhonghua 11 plants were grown in a greenhouse with a 14-h light/10-h dark cycle. MeJA treatment was conducted by spraying $0.2 \mathrm{mM}$ MeJA on the leaves. Five plants were collected at each time point of $0,1,2,3$ and $12 \mathrm{~h}$ after treatment for RNA isolation.

For MeJA treatment at the seedling stage, $\mathrm{T}_{2}$ seeds of Zhonghua 11 and overexpression lines were sterilized and germinated on 1/2 MS medium with 16-h light/8-h dark cycle for 4 days, and then transferred to $1 / 2 \mathrm{MS}$ medium containing $25 \mu \mathrm{M}$ MeJA. The shoot and root lengths of the seedlings were measured after 10 days of growth.

\section{Subcellular localization of OsNAP}

The cDNA fragment of OsNAP was amplified using the full-length cDNA clone J100075D15 (http://cdna01.dna. affrc.go.jp/cDNA) as a template with the following primers: 5'- ATCggtaccATGGTTCTGTCGAACCCGGC-3' and 5'-ATCggatccGTTCATCCCCATGTTAGAGT. The PCR products were sub-cloned into the pU1391a-GFP vector digested with Kpn I and $B a m H$ I to form pU1391a-OsNAP-GFP [48]. The construct was transformed into onion (Allium cepa L.) epidermal cells using a Biolistic PDS-1000/He particle delivery system (Bio-Rad) according to a previously described method [48]. After fixation in $4 \%$ PFA and staining with $10 \mu \mathrm{g} / \mathrm{ml}$ DAPI, transient expression of GFP fluorescence was observed using a Confocal Laserscanning Microscope (TCS SP2, Leica, Germany).

A 1176-bp cDNA fragment of OsNAP was amplified using the following primers: 5'-gaattcATGGTTCTGTC GAACCCGGC-3' and 5'-tctagaGTTCATCCCCATGTT AGAGT-3' with a EcoR I site and a Xba I site. The PCR products were digested with $E c o R$ I/Xba I and inserted into pM999-35S-EGFP. The OsNAP-EGFP construct and a positive control construct $35 \mathrm{~S}:$ :Ghd7::CFP containing a marker gene were co-introduced into Arabidopsis protoplasts as previously described [49]. The GFP and CFP fluorescences were visualized using Confocal Laserscanning Microscopy (TCS SP2, Leica, Germany).

\section{Functional characterization of OsNAP in yeast}

For the yeast one-hybrid assay, the cDNA fragment of OsNAP was amplified and cloned into the pGADT7 (Clontech, Palo Alto, CA, USA) vector to form pGADOsNAP. pGAD-OsNAP/pHIS2-cis, the positive control (pHIS53/pGAD-Rec2-53) and the negative control (pHIS2/pGAD-OsNAP) were co-transformed into the Y187 yeast strain for determination of the DNAprotein interactions according to a previous report [34]. For the transactivation assay, the PCR product of the full-length and partial fragments of OsNAP were fused in frame to the GAL4 DNA binding domain in the pGBDK7 vector to form pGBD-OsNAP, pGBDOsNAP-ABC, pGBD-OsNAP-DE and pGBD-OsNAPTR. The combinations of pGBD-OsNAP/pGADT7, pGBD-OsNAP-ABC/pGADT7, pGBD-OsNAP-DE/pGADT7, pGBD-OsNAP-TR/pGADT7, the positive control (pHIS53/ pGAD-Rec2-53) and the negative control (pGBDK7/ pGADT7) were co-transformed into the AH109 yeast strain for the verification of transactivation activity.

\section{Construction of expression plasmids and transformation into rice}

To investigate the expression pattern of OsNAP, the promoter fragment of OsNAP was amplified using the following primers: 5'- ATCGaagcttCCGTTGCATTAGGAA ACGTC-3' and 5' -ATCGtctagaACCCACACACAACAC ACACC-3' and then cloned into the DX2181b vector [50], yielding the OsNAP promoter::GUS construct. To create the overexpression construct, the OsNAP genomic DNA sequence was amplified using the following primers: 5'- ATCGccatggTTCGCCATGTGCAATTATG 
T-3' and 5'-ATCGggttaccCAGGGAGGTGTGTGTTG TGT-3'. The PCR fragments were digested with Nco I and BstE II and cloned into the pCAMBIA1301 expression vector. To suppress the OsNAP gene, a cDNA fragment of OSNAP was amplified using the following primers:5'- ATCggatccCCACCACCAACAACAACAAC3' and 5'-ATCggtaccCTCAGTCCCAGTGACGATCC-3' and inserted into pMCG161 and pDS1301 [51] to form pMCG161-OsNAP and pDS1301-OsNAP, respectively. The pMCG161-OsNAP plasmid was digested with $S a c$ I/Spe I and inserted into the pDS1301-OsNAP to generate the RNAi construct. All the constructs were introduced into the EHA105 Agrobacterium tumefaciens strain and then introduced into the rice callus of Zhonghua 11 according to a previous method [52].

\section{Molecular analysis of putative transgenic plants}

The putative transgenic plants were identified by PCR according to a previously described method [53]. The copy numbers of the transgenic plants were confirmed by Southern blot using a published protocol [54]. The transcripts of OsNAP in single-copy transgenic lines were detected by Northern blot as described previously [2]. The probes for Southern and Northern blot were amplified using PCR with gene-specific primers listed in Additional file 1: Table S1. The PCR program was as follows: $94^{\circ} \mathrm{C}$ for $5 \mathrm{~min}$, followed by 30 cycles of $94^{\circ} \mathrm{C}$ for $30 \mathrm{~s}, 58^{\circ} \mathrm{C}$ for $30 \mathrm{~s}$, and $72^{\circ} \mathrm{C}$ for $30 \mathrm{~s}$, and finally $72^{\circ} \mathrm{C}$ for $7 \mathrm{~min}$.

\section{GUS staining assay}

GUS staining and observation were carried out as described previously [50]. Various tissues and organs, including the callus, leaves, sheaths, nodes and mature seeds from $P_{O S N A P}:: G U S$ transgenic plants were immersed in GUS staining solution $(0.1 \%$ Triton X-100, $1 \mathrm{mg} / \mathrm{mL}$ X-Gluc, $100 \mu \mathrm{g} / \mathrm{ml}$ chloramphenicol, $1 \mathrm{mM}$ potassium ferricyanide, $1 \mathrm{mM}$ potassium ferrocyanide, $10 \mathrm{mM}$ $\mathrm{Na}_{2}$-EDTA, $20 \%$ methanol and $50 \mathrm{mM}$ sodium phosphate, $\mathrm{pH} 7.0)$ at $37^{\circ} \mathrm{C}$ overnight. After staining, the samples were bleached with $75 \%(\mathrm{v} / \mathrm{v})$ ethanol and photographed using a dissecting microscope (Leica, Germany).

\section{RNA isolation and quantitative RT-PCR analysis}

Total RNAs were extracted using Trizol reagent according to the manufacturer's protocol (Invitrogen, USA). Firststrand cDNAs were synthesized in a reaction volume of $20 \mu \mathrm{L}$ containing $3 \mu \mathrm{g}$ RNase-free DNase I treated total RNA, $0.5 \mathrm{mg}$ Oligo(dT) $)_{15}, 1 \mathrm{mM}$ dNTPs, $10 \mathrm{mM}$ dithiothreitol, and 200 units of SuperScript ${ }^{\mathrm{Tm}}$ III reverse transcriptase (Invitrogen, USA). Quantitative RT-PCR (qRT-PCR) was carried out as described previously [55]. Three replicates were performed for the analysis of each gene and for determining the relative expression levels between the repeats according to a previous report [56]. Actin was used as an internal control for normalization. The primers are listed in Additional file 1: Table S1.

\section{Measurement of chlorophyll content, net photosynthetic rate and endogenous JA levels}

Chlorophyll content was determined as described previously [57]. Net photosynthetic rate was measured from 9:00 am to 11:00 am or 3:00 pm to 5:00 pm using CIRAS2 according to the manufacturer's instructions (PP system, USA). The endogenous JA level was extracted and quantified using the previously described method [58].

\section{Additional file}

Additional file 1: Table S1. PCR primers used for this study. Table S2. Analysis of cis-elements in the promoter region of JA biosynthesis-related genes tested in this study. Figure S1. Phylogenetic analysis of AtNAP and rice NAC protein sequences. Figure S2. OsNAP is a NAC protein. Figure S3. Southern blot analysis of the OSNAP-overexpressing transgenic $\mathrm{T}_{0}$ lines. Figure S4. Northern blot analysis of the OsNAP-overexpressing transgenic lines and WT control. Figure S5. Southern blot analysis of the OSNAP RNAi To lines. Figure S6. Northern blot analysis of the OsNAP RNAi $T_{0}$ lines and $W T$ control.

\section{Abbreviations}

JA: Jasmonic acid; MeJA: Methyl jasmonate acid; ORF: Open reading frame; WT: Wild-type; 3-AT: 3-amino-1,2,4-triazole; TR domain: Transcriptional regulatory (TR) domain; DAH: Day after heading.

\section{Competing interests}

The authors declare that they have no competing interests.

\section{Authors' contributions}

$Y L$ conceived the study and acquired the funding. $L L$ provided the subcellular localization in onion. WH carried out the transactivation assay and JA quantification. $Y Z$ has done the other experiments and drafted the manuscript. FZ revised the manuscript. YL contributed to data interpretation and edited the final manuscript. All authors have read and approved the final manuscript.

\section{Acknowledgments}

This research was funded by the National High Technology Research and Development Program of China (863 Program), the National Natural Science Foundation of China and the National Program of Transgenic Variety Development of China. We are grateful Dr. Zuoxiong Liu for reading the manuscript. We also thank Huazhi Song for technical support in using confocal scanning laser microscopy (CSLM).

\section{Author details}

${ }^{1}$ National Key Laboratory of Crop Genetic Improvement and National Centre of Plant Gene Research, Huazhong Agricultural University, Wuhan, 430070, China. ${ }^{2}$ Plant Reproductive Biology, Mail Stop 5, University of California, 1 Shields Avenue, Davis, CA 95616-8780, USA.

Received: 29 April 2013 Accepted: 13 August 2013 Published: 12 September 2013

\section{References}

1. Lim PO, Kim HJ, Nam HG: Leaf senescence. Annu Rev Plant Biol 2007, 58:115-136.

2. Liu L, Zhou Y, Zhou G, Ye R, Zhao L, Li X, Lin Y: Identification of early senescence-associated genes in rice flag leaves. Plant Mol Biol 2008, $67: 37-55$. 
3. Guo Y, Gan S: Convergence and divergence in gene expression profiles induced by leaf senescence and 27 senescence-promoting hormonal, pathological and environmental stress treatments. Plant Cell Environ 2012, 35:644-655.

4. Buchanan-Wollaston V, Page T, Harrison E, Breeze E, Lim PO, Nam HG, Lin JF, Wu SH, Swidzinski J, Ishizaki K: Comparative transcriptome analysis reveals significant differences in gene expression and signalling pathways between developmental and dark/starvation-induced senescence in Arabidopsis. Plant J 2005, 42:567-585.

5. Guo Y, Cai Z, Gan S: Transcriptome of Arabidopsis leaf senescence. Plant Cell Environ 2004, 27:521-549.

6. Uauy C, Distelfeld A, Fahima T, Blechl A, Dubcovsky J: A NAC gene regulating senescence improves grain protein, zinc, and iron content in wheat. Science 2006, 314:1298-1301.

7. Kim JH, Woo HR, Kim J, Lim PO, Lee IC, Choi SH, Hwang D, Nam HG: Trifurcate feed-forward regulation of age-dependent cell death involving miR164 in Arabidopsis. Science 2009, 323:1053-1057.

8. Oh SA, Park JH, Lee Gl, Paek KH, Park SK, Nam HG: Identification of three genetic loci controlling leaf senescence in Arabidopsis thaliana. Plant J 1997, 12:527-535.

9. Yang SD, Seo PJ, Yoon HK, Park CM: The Arabidopsis NAC transcription factor VNI2 integrates abscisic acid signals into leaf senescence via the COR/RD genes. Plant Cell 2011, 23:2155-2168.

10. Guo Y, Gan S: AtNAP, a NAC family transcription factor, has an important role in leaf senescence. Plant J 2006, 46:601-612.

11. Zhang K, Gan S: An abscisic acid-AtNAP transcription factor-SAG113 protein phosphatase $2 \mathrm{C}$ regulatory chain for controlling dehydration in senescing Arabidopsis leaves. Plant Physiol 2012, 158:961-969.

12. Zhang K, Xia X, Zhang Y, Gan S: An ABA-regulated and Golgi-localized protein phosphatase controls water loss during leaf senescence in Arabidopsis. Plant J 2012, 69:667-678.

13. Ueda J, Kato J: Isolation and identification of a senescence-promoting substance from wormwood (Artemisia absinthium L.). Plant Physiol 1980, 66:246-249

14. He Y, Fukushige H, Hildebrand DF, Gan S: Evidence supporting a role of jasmonic acid in Arabidopsis leaf senescence. Plant Physiol 2002, 128:876-884.

15. Buchanan-Wollaston V, Earl S, Harrison E, Mathas E, Navabpour S, Page T, Pink D: The molecular analysis of leaf senescence - a genomics approach. Plant Biotechnol J 2003, 1:3-22.

16. van der Graaff E, Schwacke R, Schneider A, Desimone M, Flugge UI, Kunze R: Transcription analysis of Arabidopsis membrane transporters and hormone pathways during developmental and induced leaf senescence. Plant Physiol 2006, 141:776-792.

17. Robatzek S, Somssich IE: A new member of the Arabidopsis WRKY transcription factor family, AtWRKY6, is associated with both senescence- and defence-related processes. Plant J 2001, 28:123-133.

18. Lee RH, Hsu JH, Huang HJ, Lo SF, Chen SC: Alkaline alpha-galactosidase degrades thylakoid membranes in the chloroplast during leaf senescence in rice. New Phytol 2009, 184:596-606.

19. Miao Y, Zentgraf U: The antagonist function of Arabidopsis WRKY53 and ESR/ESP in leaf senescence is modulated by the jasmonic and salicylic acid equilibrium. Plant Cell 2007, 19:819-830.

20. Lee RH, Lin MC, Chen SC: A novel alkaline alpha-galactosidase gene is involved in rice leaf senescence. Plant Mol Biol 2004, 55:281-295.

21. Hung $\mathrm{KT}, \mathrm{Hsu} \mathrm{YT}$, Kao $\mathrm{CH}$ : Hydrogen peroxide is involved in methyl jasmonate-induced senescence of rice leaves. Physiol Plant 2006, 127:293-303.

22. Ooka H, Satoh K, Doi K, Nagata T, Otomo Y, Murakami K, Matsubara K, Osato $\mathrm{N}$, Kawai J, Carninci P: Comprehensive analysis of NAC family genes in Oryza sativa and Arabidopsis thaliana. DNA Res 2003, 10:239-247.

23. Fang $Y$, You J, Xie K, Xie W, Xiong L: Systematic sequence analysis and identification of tissue-specific or stress-responsive genes of NAC transcription factor family in rice. Mol Genet Genomics 2008, 280:547-563.

24. Nuruzzaman M, Manimekalai R, Sharoni AM, Satoh K, Kondoh H, Ooka H, Kikuchi S: Genome-wide analysis of NAC transcription factor family in rice. Gene 2010, 465:30-44

25. Wang L, Xie W, Chen Y, Tang W, Yang J, Ye R, Liu L, Lin Y, Xu C, Xiao J, Zhang Q: A dynamic gene expression atlas covering the entire life cycle of rice. Plant J 2010, 61:752-766.

26. Sato Y, Antonio BA, Namiki N, Takehisa H, Minami H, Kamatsuki K, Sugimoto K, Shimizu Y, Hirochika H, Nagamura Y: RiceXPro: a platform for monitoring gene expression in japonica rice grown under natural field conditions. Nucleic Acids Res 2011, 39:D1141-D1148.
27. Sperotto RA, Ricachenevsky FK, Duarte GL, Boff T, Lopes KL, Sperb ER, Grusak MA, Fett JP: Identification of up-regulated genes in flag leaves during rice grain filling and characterization of OsNAC5, a new ABAdependent transcription factor. Planta 2009, 230:985-1002.

28. Nakashima K, Tran LSP, Van Nguyen D, Fujita M, Maruyama K, Todaka D, Ito Y, Hayashi N, Shinozaki K, Yamaguchi-Shinozaki K: Functional analysis of a NACtype transcription factor OsNAC6 involved in abiotic and biotic stressresponsive gene expression in rice. Plant J 2007, 51:617-630.

29. Takasaki H, Maruyama K, Kidokoro S, Ito Y, Fujita Y, Shinozaki K, YamaguchiShinozaki K, Nakashima K: The abiotic stress-responsive NAC-type transcription factor OsNAC5 regulates stress-inducible genes and stress tolerance in rice. Mol Genet Genomics 2010, 284:173-183.

30. Hu H, You J, Fang Y, Zhu X, Qi Z, Xiong L: Characterization of transcription factor gene SNAC2 conferring cold and salt tolerance in rice. Plant $\mathrm{Mol}$ Biol 2008, 67:169-181.

31. Kikuchi K, Ueguchi-Tanaka M, Yoshida K, Nagato Y, Matsusoka M, Hirano HY: Molecular analysis of the NAC gene family in rice. Mol Genet Genomics 2000, 262:1047-1051

32. Puranik S, Sahu PP, Srivastava PS, Prasad M: NAC proteins: regulation and role in stress tolerance. Trends Plant Sci 2012, 17:369-381.

33. Xue W, Xing Y, Weng X, Zhao Y, Tang W, Wang L, Zhou H, Yu S, Xu C, Li X: Natural variation in Ghd7 is an important regulator of heading date and yield potential in rice. Nature Genet 2008, 40:761-767.

34. Hu H, Dai M, Yao J, Xiao B, Li X, Zhang Q, Xiong L: Overexpressing a NAM, ATAF, and CUC (NAC) transcription factor enhances drought resistance and salt tolerance in rice. Proc Natl Acad Sci USA 2006, 103:12987-12992.

35. Tran LSP, Nakashima K, Sakuma Y, Simpson SD, Fujita Y, Maruyama K, Fujita $M$, Seki M, Shinozaki K, Yamaguchi-Shinozaki K: Isolation and functional analysis of Arabidopsis stress-inducible NAC transcription factors that bind to a drought-responsive cis-element in the early responsive to dehydration stress 1 promoter. Plant Cell 2004, 16:2481-2498.

36. Fujita M, Fujita Y, Maruyama K, Seki M, Hiratsu K, Ohme-Takagi M, Tran LSP, Yamaguchi-Shinozaki K, Shinozaki K: A dehydration-induced NAC protein, $\mathrm{RD} 26$, is involved in a novel ABA-dependent stress-signaling pathway. Plant J 2004, 39:863-876.

37. Kong Z, Li M, Yang W, Xu W, Xue Y: A novel nuclear-localized CCCH-type zinc finger protein, OsDOS, is involved in delaying leaf senescence in rice. Plant Physiol 2006, 141:1376-1388.

38. Olsen AN, Ernst HA, Leggio LL, Skriver K: NAC transcription factors: structurally distinct, functionally diverse. Trends Plant Sci 2005, 10:79-87.

39. Balazadeh S, Siddiqui H, Allu AD, Matallana-Ramirez LP, Caldana C, Mehrnia M, Zanor MI, Kohler B, Mueller-Roeber B: A gene regulatory network controlled by the NAC transcription factor ANAC092/AtNAC2/ORE1 during saltpromoted senescence. Plant J 2010, 62:250-264.

40. Wasternack C: Jasmonates: an update on biosynthesis, signal transduction and action in plant stress response, growth and development. Ann Bot 2007, 100:681-697.

41. Reinbothe C, Springer A, Samol I, Reinbothe S: Plant oxylipins: role of jasmonic acid during programmed cell death, defence and leaf senescence. FEBS J 2009, 276:4666-4681.

42. Wasternack C, Hause B: Jasmonates: biosynthesis, perception, signal transduction and action in plant stress response, growth and development. An update to the 2007 review in Annals of Botany. Ann Bot 2013, 111:1021-1058.

43. Tsuchiya T, Ohta H, Okawa K, Iwamatsu A, Shimada H, Masuda T, Takamiya K-i: Cloning of chlorophyllase, the key enzyme in chlorophyll degradation: finding of a lipase motif and the induction by methyl jasmonate. Proc Natl Acad Sci USA 1999, 96:15362-15367.

44. He Y, Tang W, Swain JD, Green AL, Jack TP, Gan S: Networking senescenceregulating pathways by using Arabidopsis enhancer trap lines. Plant Physiol 2001, 126:707-716.

45. Xiao S, Dai L, Liu F, Wang Z, Peng W, Xie D: COS1: an Arabidopsis coronatine insensitive 1 suppressor essential for regulation of jasmonate-mediated plant defense and senescence. Plant Cell 2004, 16:1132-1142.

46. Shan X, Wang J, Chua L, Jiang D, Peng W, Xie D: The role of Arabidopsis rubisco activase in jasmonate-induced leaf senescence. Plant Physiol 2011, 155:751-764.

47. Schommer C, Palatnik JF, Aggarwal P, Chetelat A, Cubas P, Farmer EE, Nath U, Weigel D: Control of jasmonate biosynthesis and senescence by miR319 targets. PLoS Biol 2008, 6:e230. 
48. Huang $L$, Sun $Q$, Qin F, Li C, Zhao Y, Zhou DX: Down-regulation of a SILENT INFORMATION REGULATOR2-related histone deacetylase gene, OSSRT1, induces DNA fragmentation and cell death in rice. Plant Physiol 2007, 144:1508-1519.

49. Du H, Liu L, You L, Yang M, He Y, Li X, Xiong L: Characterization of an inositol 1, 3, 4-trisphosphate 5/6-kinase gene that is essential for drought and salt stress responses in rice. Plant Mol Biol 2011, 77:547-563.

50. Ye R, Zhou F, Lin Y: Two novel positive cis-regulatory elements involved in green tissue-specific promoter activity in rice (Oryza sativa $L \mathrm{ssp}$.). Plant Cell Rep 2012, 31:1159-1172.

51. Yuan B, Shen X, Li X, Xu C, Wang S: Mitogen-activated protein kinase OsMPK6 negatively regulates rice disease resistance to bacterial pathogens. Planta 2007, 226:953-960.

52. Hiei Y, Ohta S, Komari T, Kumashiro T: Efficient transformation of rice (Oryza sativa L.) mediated by Agrobacterium and sequence analysis of the boundaries of the T-DNA. Plant J 1994, 6:271-282.

53. Liu L, Zhou Y, Szczerba MW, Li X, Lin Y: Identification and application of a rice senescence-associated promoter. Plant Physiol 2010, 153:1239-1249.

54. Nadal A, Montero M, Badosa E, Messeguer J, Montesinos L, Montesinos E, Pla M: Constitutive expression of transgenes encoding derivatives of the synthetic antimicrobial peptide BP100: impact on rice host plant fitness. BMC Plant Biology 2012, 12:159-180.

55. Qiu D, Xiao J, Xie W, Cheng H, Li X, Wang S: Exploring transcriptional signalling mediated by OsWRKY13, a potential regulator of multiple physiological processes in rice. BMC Plant Biology 2009, 9:74-85.

56. Livak KJ, Schmittgen TD: Analysis of relative gene expression data using real-time quantitative PCR and the 2-[Delta][Delta] CT method. Methods 2001, 25:402-408.

57. Mao D, Yu H, Liu T, Yang G, Xing Y: Two complementary recessive genes in duplicated segments control etiolation in rice. Theor App/ Genet 2011, 122:373-383.

58. Ding $X, C a o$ Y, Huang L, Zhao J, Xu C, Li X, Wang S: Activation of the indole-3-acetic acid-amido synthetase $\mathrm{GH} 3-8$ suppresses expansin expression and promotes salicylate-and jasmonate-independent basal immunity in rice. Plant Cell 2008, 20:228-240.

doi:10.1186/1471-2229-13-132

Cite this article as: Zhou et al.: Identification and functional characterization of a rice NAC gene involved in the regulation of leaf senescence. BMC Plant Biology 2013 13:132.

\section{Submit your next manuscript to BioMed Central and take full advantage of:}

- Convenient online submission

- Thorough peer review

- No space constraints or color figure charges

- Immediate publication on acceptance

- Inclusion in PubMed, CAS, Scopus and Google Scholar

- Research which is freely available for redistribution 